\title{
ARTICLE
}

\section{Chronic myelogenous leukemia \\ Defining therapy goals for major molecular remission in chronic myeloid leukemia: results of the randomized CML Study IV}

\author{
Susanne Saussele ${ }^{1} \cdot$ Rüdiger Hehlmann $^{1} \cdot$ Alice Fabarius $^{1} \cdot$ Sabine Jeromin $^{2}$ • Ulrike Proetel ${ }^{1}$ - Sebastien Rinaldetti ${ }^{1}$. \\ Katharina Kohlbrenner ${ }^{1} \cdot$ Hermann Einsele $^{3} \cdot$ Christiane Falge $^{4} \cdot$ Lothar Kanz $^{5}$ - Andreas Neubauer ${ }^{6}$.

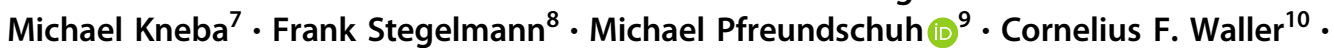 \\ Elisabeth Oppliger Leibundgut ${ }^{11} \cdot$ Dominik Heim $^{12} \cdot$ Stefan W. Krause ${ }^{13} \cdot$ Wolf-Karsten Hofmann $^{1} \cdot$ Joerg Hasford $^{14}$. \\ Markus Pfirrmann ${ }^{14} \cdot$ Martin C. Müller $^{1}$. Andreas Hochhaus ${ }^{15} \cdot$ Michael Lauseker $^{14}$
}

Received: 8 November 2017 / Revised: 8 January 2018 / Accepted: 15 January 2018 / Published online: 26 February 2018

(c) The Author(s) 2018. This article is published with open access

\begin{abstract}
Major molecular remission (MMR) is an important therapy goal in chronic myeloid leukemia (CML). So far, MMR is not a failure criterion according to ELN management recommendation leading to uncertainties when to change therapy in CML patients not reaching MMR after 12 months. At monthly landmarks, for different molecular remission status Hazard ratios (HR) were estimated for patients registered to CML study IV who were divided in a learning and a validation sample. The minimum HR for MMR was found at 2.5 years with 0.28 (compared to patients without remission). In the validation sample, a significant advantage for progression-free survival (PFS) for patients in MMR could be detected ( $p$-value 0.007). The optimal time to predict PFS in patients with MMR could be validated in an independent sample at 2.5 years. With our model we provide a suggestion when to define lack of MMR as therapy failure and thus treatment change should be considered. The optimal response time for 1\% BCR-ABL at about 12-15 months was confirmed and for deep molecular remission no specific time point was detected. Nevertheless, it was demonstrated that the earlier the MMR is achieved the higher is the chance to attain deep molecular response later.
\end{abstract}

Electronic supplementary material The online version of this article (https://doi.org/10.1038/s41375-018-0055-7) contains supplementary material, which is available to authorized users.

Susanne Saussele

susanne.saussele@medma.uni-heidelberg.de

1 III. Medizinische Klinik, Universitätsmedizin Mannheim, Universität Heidelberg, Mannheim, Germany

2 MLL Münchner Leukämielabor, München, Germany

3 Medizinische Klinik II, Universitätsklinikum, Würzburg, Germany

4 Medizinische Klinik 5, Klinikum Nord, Nürnberg, Germany

5 Innere Medizin II, Universitätsklinikum, Tübingen, Germany

6 Klinik für Hämatologie, Zentrum Innere Medizin, PhilippsUniversität, Marburg, Germany

7 II. Medizinische Klinik und Poliklinik im SKK, Universitätsklinikum Schleswig-Holstein, Kiel, Germany

8 Klinik für Innere Medizin III, Universitätsklinikum, Ulm, Germany

\section{Introduction}

Since the introduction of tyrosine kinase inhibitors (TKI) survival in chronic myeloid leukemia (CML) has profoundly improved. Inside clinical trials, survival of CML patients is comparable to that of the general population [1].

9 Klinik für Innere Medizin I, Universitätsklinikum des Saarlandes, Homburg/Saar, Germany

10 Klinik für Innere Medizin I, Universitätsklinikum, Freiburg, Germany

11 Universitätsklinik für Hämatologie und hämatologisches Zentrallabor, Inselspital, Bern, Switzerland

12 Klinik für Hämatologie, Universitätsspital, Basel, Switzerland

13 Medizinische Klinik 5, Universitätsklinikum, Erlangen, Germany

14 Institut für Medizinische Informationsverarbeitung, Biometrie und Epidemiologie, Ludwig-Maximilians-Universität, München, Germany

15 Klinik für Innere Medizin II, Abt. Hämatologie und Intern. Onkologie, Universitätsklinikum, Jena, Germany 
Comorbidities have more influence on survival than CML itself [2, 3]. Achieving complete cytogenetic remission (CCyR) is a well-accepted milestone in the management of CML [4]. In the management recommendations of the European LeukemiaNet (ELN), achievement of CCyR is included in the criteria for optimal response, warning and failure in the course of the disease [5]. However, the optimal time to wait for major molecular remission (MMR) is unknown. One study could show a significant impact of MMR at 12 month on overall survival (OS) [6, 7]. However, if patients are divided in response levels $>1,0.1-1$ and $<0.1 \%$ the significance between the latter groups is no longer detectable. Other studies could not find an effect of MMR on OS at all $[8,9]$. Therefore, failure to achieve an MMR has not been defined as a failure criterion in the ELN recommendations.

Under TKI therapy, the majority of CML patients reaches major or even deep molecular remissions [10,11], which are indications of a good prognosis [12]. However, a small percentage of patients do not achieve MMR. The question is: when is it necessary to regard a lack of MMR as "failure" and to switch therapy. Therefore, we were looking for a critical turning point at which waiting for an MMR is no longer promising. The same analysis was conducted for BCR-ABL levels $<1 \%\left(\mathrm{MR}^{2}\right)$ as well. In addition, potential milestones for the achievement of MR4.5 were investigated.

We used data of the CML study IV to estimate in a mathematical model the time to define a treatment failure in a CML patient without MMR under imatinib therapy.

\section{Patients and methods}

\section{Study design and goals}

From July 2002 through March 2012, 1551 patients were recruited for the CML study IV, a five-arm randomized trial for chronic-phase CML comparing first-line imatinib treatment with different dosages and with or without additional non-TKI therapy.

The study design and patient characteristics have been described in detail $[6,7] .1228$ patients were available for the current analyses after exclusion of 128 patients from the "Imatinib after IFN"-arm, 164 with molecular analyses in non-standardized laboratories resp. not sufficient for the detection of a MR ${ }^{4}, 16$ without EUTOS score, and 15 due to various other reasons (see Fig. 1, Consort diagram). The median age of the analyzed patients was 52 years (range: $16-85) ; 742(60.4 \%)$ of the patients were male and $1080(88 \%)$ were low-risk patients according to the EUTOS score. The median observation time was 7.0 years.

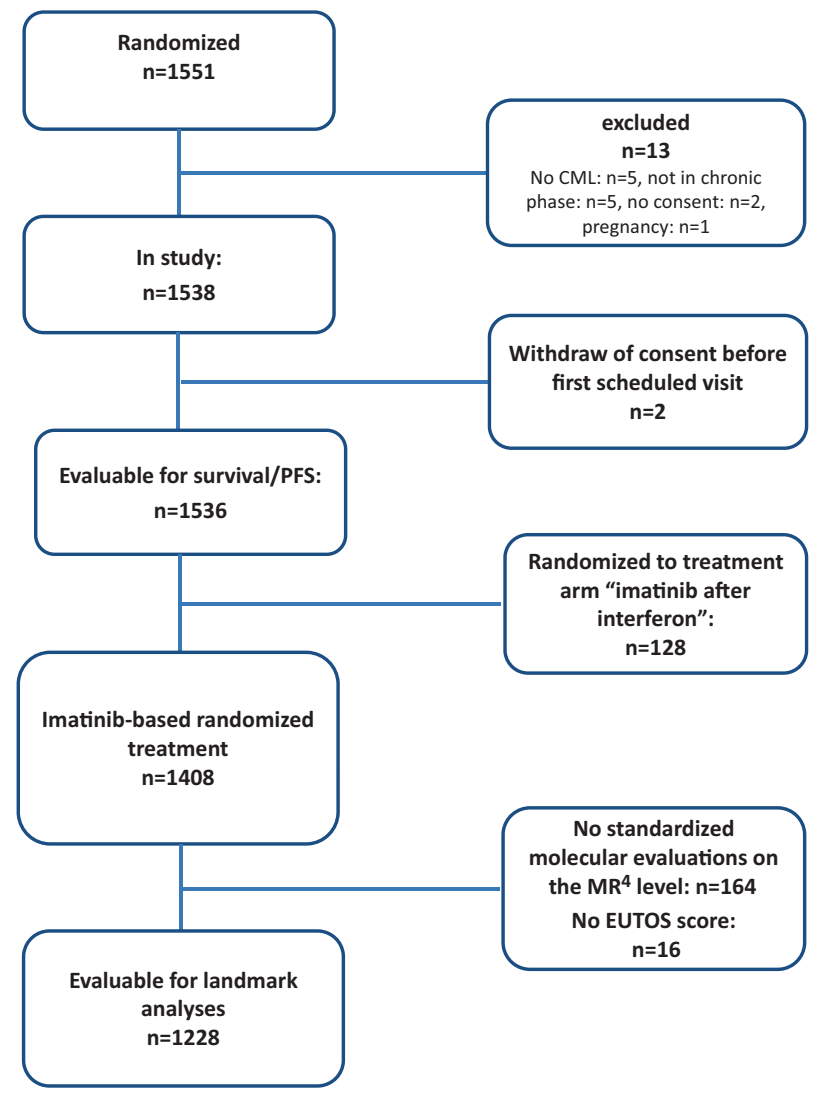

Fig. 1 Consort diagram

\section{Definitions}

Progression-free survival (PFS) was defined as survival with the absence of accelerated phase, blast crisis or death. PFS times and times to $\mathrm{MR}^{4.5}$ were calculated starting at the date of diagnosis. For $\mathrm{MR}^{4.5}$, progression or death were considered competing events. Patients without an event were censored at the date of the last observation. Patients with stem cell transplantation were censored at the date of transplantation.

Patients were considered to be in $\mathrm{MR}^{2}$, when they had achieved a BCR-ABL/ABL ratio of less than $1 \%$ according to the international scale (IS) [13, 14], which can be regarded as an equivalent to complete cytogenetic remission [15]. MMR was defined as a BCR-ABL/ABL (IS) value below $0.1 \%$, while $\mathrm{MR}^{4}$ and $\mathrm{MR}^{4.5}$ were defined as BCRABL/ABL (IS) $<0.01 \%$ and $<0.0032 \%$, respectively.

\section{Cytogenetic and molecular analyses}

Molecular diagnostics for residual BCR-ABL transcripts followed the procedures and definitions of Hughes et al. [16] and Cross et al. [13] and were performed in standardized and accredited laboratories with defined 
conversion factors for the equivalence of the tests (Mannheim, Basel, Bern and MLL Munich) [12, 13]. Median molecular examinations per year were 2.9 per patient.

\section{Statistics}

Patients were randomly divided into a learning and a validation set at a ratio of $2: 1$.

To get a function of the hazard ratio (HR) depending on the prediction time, we used the landmarking approach of van Houwelingen: [17, 18] Starting at 6 months after diagnosis, at each month until month 60 , patients of the learning sample still at risk were categorized into three groups ("no remission", "MR ${ }^{2}$ " and "MMR or deeper") according to their deepest remission achieved until that time. The state "No remission" was defined as not even having reached an $\mathrm{MR}^{2}$. Patients that were switched to any second-generation TKI were counted as "no remission"or " $\mathrm{MR}^{2}$ " after the switch, if they had not reached an $\mathrm{MR}^{2}$ or MMR before switch, respectively. 106 patients in the learning sample were switched to a $2^{\text {nd }}$-generation TKI before achieving a MMR. A Cox model was fitted to the combined data set at all landmarks where the baseline hazard as well as the regression coefficients of the timevarying remission states were dependent on the landmark time. This time-dependence was modeled using a cubic function of time. In addition, EUTOS score [4, 19] and age were considered as covariates for adjustment. Details can be found in the online supplement.

To account for the heterogeneity in the data set and to receive stable confidence intervals, bootstrap resampling was used and the procedure was repeated 10,000 times. Out of these 10,000 estimates of the regression coefficient functions, a median curve was calculated (together with $95 \%$ confidence intervals). The minimum of this median curve is the point, where the largest difference between the patients in remission and those without was found. Therefore it was considered as cutoff.

For the validation, a bootstrap was performed again. In the 10,000 random subsets from the validation set, patients were categorized into "no remission", "MR" and "MMR (or deeper)" at the cutoff derived from the learning sample.

All computations were performed using the statistics software R 3.0.2.

\section{Ethics}

The protocol followed the Declaration of Helsinki and was approved by the local ethics committees. Written informed consent was obtained from all patients before they entered the study.

\section{Results}

\section{Learning set}

Two thirds of the total data set ( $n=819$ patients) were randomly allocated to the learning sample. At 6 months, 805 patients were still alive and without progression. Of those, $534(66 \%)$ had no remission, $182(23 \%)$ had achieved an $\mathrm{MR}^{2}$ and $89(11 \%)$ an MMR. Naturally, the percentage of patients without molecular remission is decreasing over time, while the percentage of patients with MMR is increasing. The total number of patients is decreasing, since patients who had a progression are not in the data set at a later landmark anymore, nor are patients with a shorter observation time. At the last landmark at 5 years, 528 patients were still at risk. This is depicted in Fig. 2.
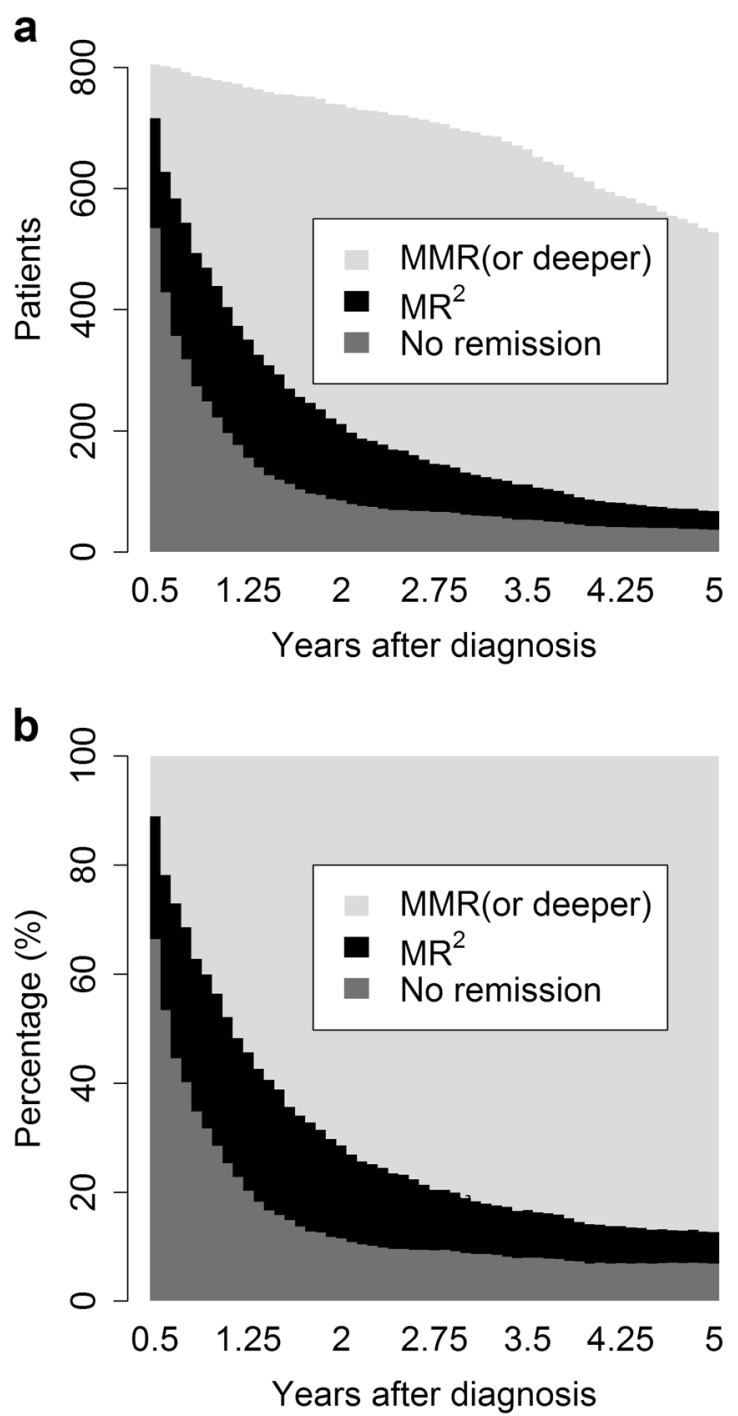

Fig. 2 Distribution of patients according to remission status achieved at different landmarks from 6 months to 5 years (a) and respective percentages of these states (b) 


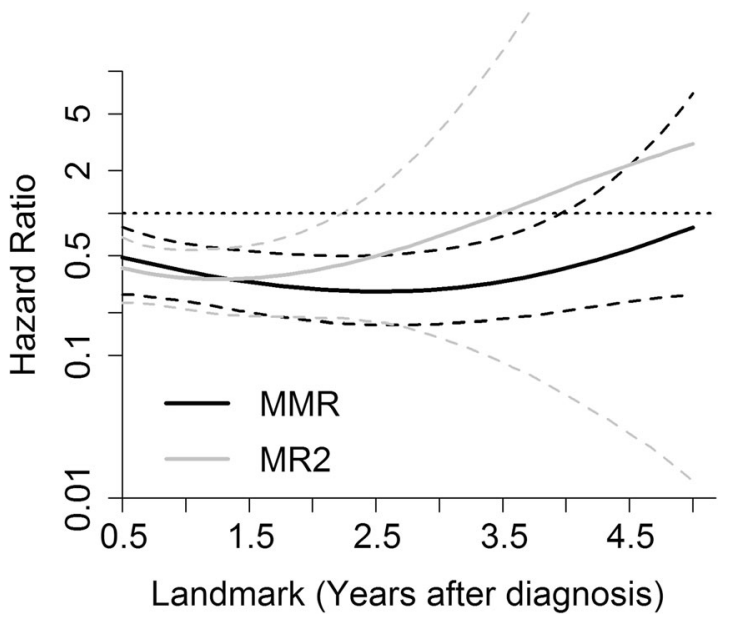

Fig. 3 Median hazard ratio functions for the comparison of patients who had achieved a MMR resp. $\mathrm{MR}^{2}$ to those who did not have any remission at different landmarks with respect to PFS from 6 months to 5 years together with the $95 \%$ confidence intervals. On the $y$ axis, the hazard ratio for PFS is plotted on a logarithmic scale. Note that on the $x$ axis the landmark time is plotted instead of the event time. A hazard ratio of, e.g., 0.5 at landmark 6 months indicates that patients with MMR have only half the risk of patients with no MMR before or at 6 months

As described in the methods section, we drew bootstrap sets (with replacement) from the learning set. For each of these 10,000 subsets, a Cox model was estimated, resulting in a HR for suffering a progression, dependent on the landmark time. From this $10,000 \mathrm{~h}$ functions, the median and $95 \%$ bootstrap confidence intervals were taken (see Supplementary figure 1). Figure 3 shows the HR for progression (y axis) dependent on the landmark time ( $x$ axis). When e.g. setting the landmark at 6 months, patients that had already achieved an MMR by this time, had only about 0.5 times the risk of those that did not. For the statistical details, we refer to the online supplement. The minimum of this HR function was found between the landmarks 2.33 and 2.75 years with a HR of $0.28(95 \%$ CI: 0.16-0.51). The model was adjusted for two additional covariates, age and EUTOS score. For the first covariate we observed HRs of 1.05 per year $(95 \% \mathrm{CI}$ : 1.02-1.08). For the comparison of high- vs. low-risk patients (EUTOS score), HRs of $1.93 \quad(95 \%$ CI: 0.89-3.77) were observed.

Analogously to the HR curve for MMR, the HR for MR ${ }^{2}$ was also estimated. This curve is depicted as well in Fig. 3. The minimum of the $\mathrm{MR}^{2}$ curve was at 1.25 years, with very similar values between 1.17 and 1.33 years. The HR in comparison with patients without any remission was 0.34 (95\% CI: $0.20-0.56$ ).

Additionally, the model was re-estimated with four different groups: "no remission", "MR2", "MMR" and "MR and deeper". In this analysis, the shape of the curve was slightly different, with a minimum for the patients in MMR

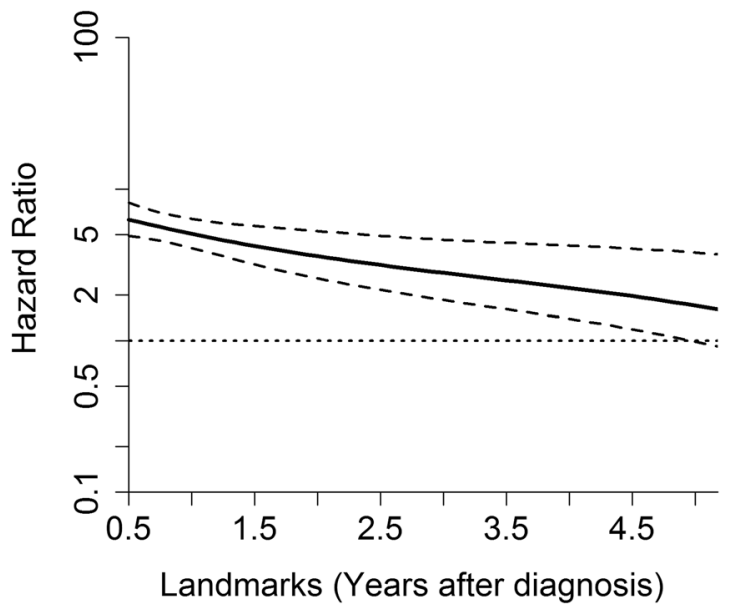

Fig. 4 Median hazard ratio function for the comparison of patients who had achieved an MMR to those who did not have any remission at different landmarks with respect to $\mathrm{MR}^{4.5}$ from 6 months to 5 years together with the $95 \%$ confidence intervals. On the $y$ axis, the hazard ratio for $\mathrm{MR}^{4.5}$ is plotted on a logarithmic scale. Note that on the $x$ axis the landmark time is plotted instead of the event time

between landmark times 3.00 and 3.92 years and an HR of 0.28 (95\% CI: $0.11-0.61$ ).

When stratifying due to imatinib (IM) dose (i.e., patients from the imatinib $800 \mathrm{mg}$ vs. patients from the three imatinib $400 \mathrm{mg}$ arms with or without other treatments), the curve for the imatinib $400 \mathrm{mg}$ arms was rather similar to the one shown in Fig. 3. For the imatinib $800 \mathrm{mg}$ patients, we did not find a minimum, the curve remained almost constant after about 2.75 years, but with much wider confidence intervals. Therefore, no separate proper cutoff could be found for this arm.

In a sensitivity analysis a similar landmark for the outcome $\mathrm{MR}^{4.5}$ instead of PFS was tried to be found (see Supplementary figure 2). The results are depicted in Fig. 4. In contrast to the PFS analysis before, we were not able to find a cutoff, the form of the function suggested that the probability of achieving $\mathrm{MR}^{4.5}$ was the higher the earlier the patient had achieved an MMR.

In our analysis, patients were always considered to be not in $\mathrm{MR}^{2}$ or MMR, respectively, when they were switched to a second-generation TKI before the achievement of these remissions. To assess the impact, we performed an additional analysis, were we ignored the switch. For the MMR we found the minimum at 2.75 years with a HR of 0.24 (95\% CI: 0.14-0.44) and similar values between 2.58 and 2.75 years. For the $\mathrm{MR}^{2}$, the minimum was at 15 months again, with a HR of 0.32 (95\% CI: 0.18-0.53) and similar values between 1.00 and 1.58 years.

\section{Validation set}

Based on the results described above, we used the landmark of 2.5 years for further analysis, because visits at 3 months 
intervals are a common practice in clinical trials. Again, 10,000 subsets were drawn out of the 409 observations of the validation set. The median HR for PFS for the patients in MMR (compared with those without remission) was 0.20 (95\% CI: 0.13-0.69). The corresponding median $p$-value was 0.007 , herewith confirming the MMR landmark of 2.5 years in predicting PFS.

We further validated the landmark of 1.25 years for the $\mathrm{MR}^{2}$. The median HR for PFS for the patients in $\mathrm{MR}^{2}$ (compared with those without remission) was 0.45 (95\% CI: $0.25-0.85)$. The corresponding median $p$-value was 0.023 . For comparison, we evaluated the established landmark of 12 months as well and found a median $p$-value of 0.047 .

\section{Discussion}

The aim of this analysis was to define treatment failure with respect to MMR. We sought to find the landmark showing the largest difference between patients in MMR and those without any remission with regard to PFS. This landmark was found at 2.5 years and was successfully validated in an independent patient sample. Therefore, this analysis is able to close the gap in the ELN recommendations for the definition of failure; a gap that existed between the nonachievement of CCyR at 12 months and the loss of MMR at any time.

It could be demonstrated for the first time, that MMR has a significant impact on PFS. In addition, we were able to replicate the 12 month landmark for the achievement of $\mathrm{MR}^{2}$, which corresponds to CCyR. Until about 1.5 years, the $\mathrm{MR}^{2}$ and the MMR curve are almost identical. This corresponds to the clinical experience that for this time period, an $\mathrm{MR}^{2}$ is sufficient in respect for PFS.

During the further course of the disease, the achievement of MMR gets more and more important. It will be of interest in the future if at some landmark even MMR is not sufficient in preventing progression.

For the prediction of the achievement of an $\mathrm{MR}^{4.5}$, we were not able to find a particular landmark. However, there was a clear correlation; the earlier MMR was reached the higher was the probability to achieve a $\mathrm{MR}^{4.5}$ during the disease course. This has to be taken into account for decision making in view of the new possibility of treatment cessation in CML [20, 21]. So far, it has not been proven that the earlier MMR and $\mathrm{MR}^{4.5}$ are reached the more successful is treatment cessation. However, the time being in MMR or deeper seems to be of relevance [22].

From our point of view, these results are of high clinical relevance as with the success of molecular standardization in Europe, molecular assessments have increasingly replaced cytogenetics. Vice versa, even with the increasing impact of deep molecular responses $\left(\mathrm{MR}^{4}\right.$ and $\left.\mathrm{MR}^{4.5}\right)$,
MMR still remains an important benchmark: (i) MMR is an indicator for deep response and (ii) there are laboratories in the daily routine care that are not able to detect deep responses correctly.

From a methodological point of view, it seems possible to censor patients receiving 2nd line TKI. However, as the loss of a remission and perhaps a subsequent progression are among the most frequent indications for switching TKI, this would have introduced a bias. Furthermore, not censoring for 2nd generation TKI should reflect the current situation in routine care. We found a minor difference for the cutoff with censoring of patients after switching TKI.

Like every other cutoff value, this one is a compromise as well. It has to be weighted between not identifying patients that would need to be switched on the one hand and identifying (and switching) too many patients that would still have achieved an MMR later on, on the other hand. Therefore, this cutoff has to be discussed again, when, e.g., new agents enter the market and the risk-benefit ratio has changed.

Our work has strengths but also some limitations. It is possible that the identified landmark is dose-dependent. Due to the low number of events in the imatinib 800 -arm, the analysis stratified for different dosages had a lack of power regarding the potential identification of a significant influence of dosage. Therefore, despite the internal validation, an external validation of the results with a completely different data set is encouraged, i.e., to check the robustness of the landmark time under different treatments. In addition, it has to be stated that this retrospective analysis was not prespecified in the protocol, which reinforces the demand for an external validation.

On the other hand this is probably the largest data set of CML patients in the imatinib era with a sufficiently long follow up under the conditions of a controlled clinical trial. The patient population of the CML IV study is-despite of the lower age-very close to cohorts of registries especially in regards to comorbidities. Therefore, we think that the results are applicable outside of trials.

It has to be stated that in first-line studies with nilotinib, dasatinib or bosutinib [23-28], molecular remission levels were achieved faster as compared with imatinib in the standard dosage of $400 \mathrm{mg}$. One can assume that in a corresponding analysis with 2nd generation TKI the recommendable time to judge on therapy failure could be less than 2.5 years as identified in this analysis.

These data show that an optimum time frame exists in order to predict PFS based on the achievement of MMR. This should be important for the optimization of treatment in CML concerning prolongation of survival. In regards of treatment cessation we have demonstrated that the earlier a patient achieved MMR the higher was the chance to reach DMR later on. 
Acknowledgements The contributions of S. Dean, E. Matzat, R. PleilLösch, I. Stalljann, G. Bartsch, U. Kossak, C. Sodan-Boyer, and U. Böhm are acknowledged. The authors thank the German Chronic Myeloid Leukemia Study Group for its participation in this study, a complete membership list appears in the Appendix. This work was supported by the Deutsche Krebshilfe (Grant 106642), Novartis (Nürnberg, Germany), Kompetenznetz für Akute und Chronische Leukämien (Bundesministerium für Bildung und Forschung 01GI0270), Deutsche José-Carreras Leukämiestiftung (Deutsche JoseCarreras-Stiftung H09/01f, H06/04v, H03/01, R05/23), European LeukemiaNet (LSHC-CT-2004-503216), Roche (Grenzach-Wyhlen, Germany), and Essex Pharma (München, Germany).This trial was registered at www.clinicaltrials.gov as \# NCT 00055874.

Authors Contributions: S.S. and M.L. had the primary responsibility for the publication. S.S., R.H., A.F., S.J., U.P., S.R., K.K., H.E., C.F., L.K., A.N., M.K, F.S., M.P., C.F.W., E.O., D.H., S.W.K., W.-K. H., J. H. M.Pfi., M.C.M., A.H. and M.L. contributed to the design of the study, to the statistical analysis and to the interpretation of the results. All authors have checked and approved the final version of the manuscript.

\section{Compliance with ethical standards}

Conflict of interest The authors declare that they have no conflict of interest. S.S.: Novartis: Honoraria, Research Funding, Travel Other; B.-M.S.: Honoraria, Research Funding, Travel, Travel Other; Pfizer: Honoraria, Travel, Travel Other. R.H.: B.-M.S.: Research Funding; Novartis: Research Funding. S.J.: MLL Munich Leukemia Laboratory: Equity Ownership. A.N.: MedUpdate: Honoraria, Speakers Bureau. M.K.: Novartis: Consultancy, Equity Ownership, Honoraria, Membership on an entity's Board of Directors or advisory committees, Research Funding. M.Pfi.: Novartis: Consultancy; B.-M.S.: Honoraria. A.H.: Novartis: Consultancy, Honoraria, Research Funding; B.-M.S.: Consultancy, Honoraria; ARIAD: Honoraria, Research Funding; Pfizer: Consultancy, Research Funding. M.C.M.: Novartis: Honoraria, Research Funding; B.-M.S.: Honoraria, Research Funding; ARIAD: Honoraria, Research Funding; Pfizer: Honoraria, Research Funding.

Open Access This article is licensed under a Creative Commons Attribution-NonCommercial-ShareAlike 4.0 International License, which permits any non-commercial use, sharing, adaptation, distribution and reproduction in any medium or format, as long as you give appropriate credit to the original author(s) and the source, provide a link to the Creative Commons license, and indicate if changes were made. If you remix, transform, or build upon this article or a part thereof, you must distribute your contributions under the same license as the original. The images or other third party material in this article are included in the article's Creative Commons license, unless indicated otherwise in a credit line to the material. If material is not included in the article's Creative Commons license and your intended use is not permitted by statutory regulation or exceeds the permitted use, you will need to obtain permission directly from the copyright holder. To view a copy of this license, visit http://creativecommons. org/licenses/by-nc-sa/4.0/.

\section{References}

1. Bower H, Bjorkholm M, Dickman PW, Hoglund M, Lambert PC, Andersson TM. Life expectancy of patients with chronic myeloid leukemia approaches the life expectancy of the general population. J Clin Oncol. 2016;34:2851-7.

2. Saussele S, Krauss MP, Hehlmann R, Lauseker M, Proetel U, Kalmanti L, et al. Impact of comorbidities on overall survival in patients with chronic myeloid leukemia: results of the randomized CML Study IV. Blood. 2015;126:42-49.

3. Pfirrmann M, Baccarani M, Saussele S, Guilhot J, Cervantes F, Ossenkoppele G, et al. Prognosis of long-term survival considering disease-specific death in patients with chronic myeloid leukemia. Leukemia. 2016;30:48-56.

4. Hasford J, Baccarani M, Hoffmann V, Guilhot J, Saussele S, Rosti $\mathrm{G}$, et al. Predicting complete cytogenetic response and subsequent progression-free survival in 2060 patients with CML on imatinib treatment: the EUTOS score. Blood. 2011;118:686-92.

5. Baccarani M, Deininger MW, Rosti G, Hochhaus A, Soverini S, Apperley JF, et al. European LeukemiaNet recommendations for the management of chronic myeloid leukemia: 2013. Blood. 2013;122:872-84.

6. Hehlmann R, Lauseker M, Jung-Munkwitz S, Leitner A, Mueller MC, Pletsch N, et al. Tolerability-adapted imatinib $800 \mathrm{mg} / \mathrm{d}$ versus $400 \mathrm{mg} / \mathrm{d}$ versus $400 \mathrm{mg} / \mathrm{d}$ plus interferon-alpha in newly diagnosed chronic myeloid leukemia. J Clin Oncol. 2011;29:1634-42.

7. Hehlmann R, Lauseker M, Saußele S, Pfirrmann M, Krause SW, Kolb H-J, et al. Assessment of imatinib as first-line treatment of chronic myeloid leukemia: 10-year survival results of the randomized CML study IV and impact of non-CML determinants. Leukemia. 2017;31:2398-406.

8. Hughes TP, Hochhaus A, Branford S, Müller MC, Kaeda JS, Foroni L, et al. Long-term prognostic significance of early molecular response to imatinib in newly diagnosed chronic myeloid leukemia: an analysis from the International Randomized Study of Interferon and STI571 (IRIS). Blood. 2010;116:3758-65.

9. Falchi L, Kantarjian HM, Wang X, Verma D, Quintas-Cardama A, O'Brien S, et al. Significance of deeper molecular responses in patients with chronic myeloid leukemia in early chronic phase treated with tyrosine kinase inhibitors. Am J Hematol. 2013;88:1024-9.

10. Kalmanti L, Saussele S, Lauseker M, Muller MC, Dietz CT, Heinrich L, et al. Safety and efficacy of imatinib in CML over a period of 10 years: data from the randomized CML-study IV. Leukemia. 2015;29:1123-32.

11. Druker BJ, Guilhot F, O'Brien SG, Gathmann I, Kantarjian H, Gattermann N, et al. Five-year follow-up of patients receiving imatinib for chronic myeloid leukemia. $N$ Engl $J$ Med. 2006;355:2408-17.

12. Hehlmann R, Müller MC, Lauseker M, Hanfstein B, Fabarius A, Schreiber A, et al. Deep molecular response is reached by the majority of patients treated with imatinib, predicts survival, and is achieved more quickly by optimized high-dose imatinib: results from the randomized CML-study IV. J Clin Oncol. 2014;32:415-23.

13. Cross NCP, White HE, Müller MC, Saglio G, Hochhaus A. Standardized definitions of molecular response in chronic myeloid leukemia. Leukemia. 2012;26:2172-5.

14. Müller MC, Cross NCP, Erben P, Schenk T, Hanfstein B, Ernst T, et al. Harmonization of molecular monitoring of CML therapy in Europe. Leukemia. 2009;23:1957-63.

15. Lauseker M, Hanfstein B, Haferlach C, Schnittger S, Pfirrmann M, Fabarius A, et al. Equivalence of BCR-ABL transcript levels with complete cytogenetic remission in patients with chronic myeloid leukemia in chronic phase. J Cancer Res Clin Oncol. 2014;140:1965-9.

16. Hughes $T$, Deininger $M$, Hochhaus A, Branford S, Radich J, Kaeda J, et al. Monitoring CML patients responding to treatment with tyrosine kinase inhibitors: review and recommendations for harmonizing current methodology for detecting BCR-ABL transcripts and kinase domain mutations and for expressing results. Blood. 2006;108:28-37. 
17. Van Houwelingen HC. Dynamic prediction by landmarking in event history analysis. Scand J Stat. 2007;34:70-85.

18. Van Houwelingen HC, Putter H. Dynamic predicting by landmarking as an alternative for multi-state modeling: an application to acute lymphoid leukemia data. Lifetime Data Anal. 2008; 14:447-63. -463

19. Hoffmann VS, Baccarani M, Lindoerfer D, Castagnetti F, Turkina A, Zaritsky A, et al. The EUTOS prognostic score: review and validation in 1288 patients with CML treated frontline with imatinib. Leukemia. 2013;27:2016-22.

20. Mahon FX, Rea D, Guilhot J, Guilhot F, Huguet F, Nicolini F, et al. Discontinuation of imatinib in patients with chronic myeloid leukaemia who have maintained complete molecular remission for at least 2 years: the prospective, multicentre Stop Imatinib (STIM) trial. Lancet Oncol. 2010;11:1029-35.

21. Saussele S, Richter J, Hochhaus A, Mahon FX. The concept of treatment-free remission in chronic myeloid leukemia. Leukemia. 2016;30:1638-47.

22. Ross DM, Branford S, Seymour JF, Schwarer AP, Arthur C, Yeung DT, et al. Safety and efficacy of imatinib cessation for CML patients with stable undetectable minimal residual disease: results from the TWISTER study. Blood. 2013;122:515-22.

23. Hughes TP, Saglio G, Kantarjian HM, Guilhot F, Niederwieser D, Rosti G, et al. Early molecular response predicts outcomes in patients with chronic myeloid leukemia in chronic phase treated with frontline nilotinib or imatinib. Blood. 2013;123:1353-60.

24. Hochhaus A, Rosti G, Cross NC, Steegmann JL, le Coutre P, Ossenkoppele G, et al. Frontline nilotinib in patients with chronic myeloid leukemia in chronic phase: results from the European ENEST1st study. Leukemia. 2015;30:57-64.

25. Firwana B, Sonbol MB, Diab M, Raza S, Hasan R, Yousef I, et al. Tyrosine kinase inhibitors as a first-line treatment in patients with newly diagnosed chronic myeloid leukemia in chronic phase: A mixed-treatment comparison. Int J Cancer. 2016;138:1545-53.

26. Hjorth-Hansen H, Stenke L, Soderlund S, Dreimane A, Ehrencrona $\mathrm{H}$, Gedde-Dahl T, et al. Dasatinib induces fast and deep responses in newly diagnosed chronic myeloid leukaemia patients in chronic phase: clinical results from a randomised phase-2 study (NordCML006). Eur J Haematol. 2015;94:243-50.

27. Jabbour E, Kantarjian HM, Saglio G, Steegmann JL, Shah NP, Boque $\mathrm{C}$, et al. Early response with dasatinib or imatinib in chronic myeloid leukemia: 3-year follow-up from a randomized phase 3 trial (DASISION). Blood. 2014;123:494-500.

28. Brummendorf TH, Cortes JE, de Souza CA, Guilhot F, Duvillie L, Pavlov D, et al. Bosutinib versus imatinib in newly diagnosed chronic-phase chronic myeloid leukaemia: results from the 24month follow-up of the BELA trial. $\mathrm{Br} \mathrm{J}$ Haematol. 2015;168:69-81. 